\title{
Online condition monitoring of wind turbines through three-phase electrical signature analysis
}

\author{
Georgia Cablea, Pierre Granjon, Christophe Bérenguer and Pascal Bellemain \\ Univ. Grenoble Alpes, GIPSA-lab, F-38000 Grenoble, France \\ CNRS, GIPSA-lab, F-38000 Grenoble, France \\ \{georgia.cablea, pierre.granjon, christophe.berenguer\}@gipsa-lab.grenoble-inp.fr
}

\begin{abstract}
In the context of the KAStrion European project, a complete solution was proposed in order to monitor wind turbines. The developed solution comprises both hardware and software parts of the condition monitoring system. In terms of software, modules for vibration analysis and electrical signal analysis have been developed. The current paper presents the electrical analysis solution proposed in the context of this project. The electrical module is able to detect both mechanical and electrical faults in a wind turbine system. The goal of the mechanical fault detection using electrical signals is to confirm the faults also detected by vibration analysis, while the main focus of the module remains the detection of electrical faults. Results showing the performance of mechanical fault detection are presented using electrical signals acquired on the test-bench developed for testing the KAStrion system. Moreover, results regarding the electrical unbalance are presented using signals acquired on a three-phase transformer. The final solution has been implemented on two onshore wind turbines since the end of 2014, and online condition monitoring results are presented at the end of the paper.
\end{abstract}

\section{Introduction}

The wind energy is a growing energy market. The annual newly installed wind turbines present a generally increasing trend worldwide. Each year, more wind turbines are installed in Europe ${ }^{(1)}$, both onshore and offshore. As the energy market relies more and more on wind energy, the availability of such energy becomes critical. The reliability of wind turbines has improved in time, both through design and implementation of efficient condition monitoring systems and predictive maintenance strategies.

The KAStrion European project ${ }^{(2)}$ aims at maximizing the production time of offshore wind turbine farms. In order to achieve this goal, a condition monitoring solution is proposed such that the diagnosis of the system can be done in real time and maintenance strategies can be properly scheduled. For condition monitoring, within this project, two main approaches are taken: vibration analysis ${ }^{(3,4)}$ and three-phase electrical signature analysis. The current paper presents the three-phase electrical analysis solution proposed in the context of this project. The main goal of this method is to detect electrical faults in a wind turbine by analysing the three-phase electrical signals acquired at the output of its generator. Its second goal is to confirm possible mechanical faults present in the system by using the same electrical measurements. 
Section 2 of this paper summarises the proposed three-phase electrical analysis method. More details on this algorithm can be found in ${ }^{(5)}$. The results for mechanical fault detection are presented in Section 3, followed by the results for electrical unbalance detection presented in Section 4. Section 5 contains preliminary results on continuous condition monitoring of two real onshore wind turbines.

\section{Three-phase electrical signals analysis method}

The proposed method considers the phasor model of three-phase electrical signals as defined in Equation (1). Electrical signals mainly consist in a fundamental component of frequency $f_{0}$, with possible amplitude and frequency modulations, some harmonics and additive noise. In what follows, $\underline{x}$ denotes the phasor of either voltage or current signals and the analysis will be performed around their fundamental frequency only.

$$
\left[\begin{array}{l}
\underline{x}_{1}(t) \\
\underline{x}_{2}(t) \\
\underline{x}_{3}(t)
\end{array}\right]=\left[\begin{array}{c}
X_{1}(t) \\
X_{2}(t) e^{-j\left(\frac{2 \pi}{3}+\rho_{1}(t)\right)} \\
X_{3}(t) e^{-j\left(\frac{4 \pi}{3}+\rho_{2}(t)\right)}
\end{array}\right] e^{j 2 \pi \int_{0}^{t} f_{0}(u) \mathrm{d} u}+\left[\begin{array}{l}
n_{1}(t) \\
n_{2}(t) \\
n_{3}(t)
\end{array}\right]
$$

In equation (1), the signal on phase 1 is considered the reference signal and the quantities described around $f_{0}$ are:

- $\underline{x}_{1}(t), \underline{x}_{2}(t)$ and $\underline{x}_{3}(t)$ representing the three-phase signals around the fundamental frequency;

- $X_{1}(t), X_{2}(t)$ and $X_{3}(t)$ representing the instantaneous magnitudes of the signals and containing possible amplitude modulations;

- $\rho_{1}(t)$ and $\rho_{2}(t)$ representing the small errors in phase shifts between phase signals;

- $f_{0}(t)$ representing the instantaneous frequency of the components and possibly containing frequency modulations;

- $n_{1}(t), n_{2}(t)$ and $n_{3}(t)$ representing additive noise which will be further neglected in this paper.

Perfectly balanced three-phase electrical signals should have the same amplitude on all phases and a phase shift of $\frac{2 \pi}{3}$ between each of the phases. This corresponds to $X_{1}(t)=$ $X_{2}(t)=X_{3}(t)$ and $\rho_{1}(t)=\rho_{2}(t)=0$. Real systems also contain an inherent small amount of electrical unbalance. However, the unbalance is generally due to faults in the threephase systems (e.g. winding stator faults in rotating machines ${ }^{(6)}$ ). One way to separate any three-phase unbalanced system into balanced components is to apply the Lyon transform ${ }^{(5,7,8)}$ as in Equation (2). 


$$
\left[\begin{array}{l}
\underline{x}_{+}(t) \\
\underline{x}_{-}(t) \\
\underline{x}_{0}(t)
\end{array}\right]=\frac{2}{3}\left[\begin{array}{ccc}
1 & a & a^{2} \\
1 & a^{2} & a \\
1 & 1 & 1
\end{array}\right]\left[\begin{array}{l}
\underline{x}_{1}(t) \\
\underline{x}_{2}(t) \\
\underline{x}_{3}(t)
\end{array}\right],
$$

where:

- $a=e^{j \frac{2 \pi}{3}}$, called the Fortescue ${ }^{(9)}$ operator, represents a phase shift of $\frac{2 \pi}{3}$;

- $\underline{x}_{+}(t), \underline{x}_{-}(t)$ and $\underline{x}_{0}(t)$ are generically called instantaneous symmetrical components and are the positive-, negative- and zero-sequence components respectively, calculated around the fundamental frequency.

In Equation (2), the factor 2 has been applied in order to obtain magnitude invariant quantities. The obtained instantaneous positive sequence component contains the amount of balanced quantity in the three-phase signals at the fundamental frequency. The negativeand zero-sequence components would be null for perfectly balanced systems. If the system also contains unbalanced quantities, the values of these two symmetrical components would increase, depending on the unbalance type. The main advantage of using instantaneous symmetrical components as opposed to classical symmetrical components ${ }^{(9)}$ is that they are time-dependent by definition. Therefore, they can be directly used to define timedependent fault indicators, which finally simplifies the conception of real-time condition monitoring strategies.

While electrical faults trigger unbalance in the three-phase electrical signals, mechanical faults trigger amplitude and frequency modulations ${ }^{(10,11,12)}$. After filtering the signals around the fundamental frequency $+f_{0}{ }^{(13)}$, the instantaneous amplitude and frequency ${ }^{(14)}$ of mono-component signals can be easily extracted as in Equations (3) and (4).

$$
\begin{array}{ccc}
X_{+}(t)=\left|\underline{x}_{+}(t)\right| & X_{-}(t)=\left|\underline{x}_{-}(t)\right| \quad X_{0}(t)=\left|\underline{x}_{0}(t)\right| \\
f_{\underline{x}_{+}}(t)=\frac{1}{2 \pi} \frac{d \theta_{+}(t)}{d t} & , \text { with: } \theta_{+}(t)=\arg \left\{\underline{x}_{+}(t)\right\}
\end{array}
$$

Using the magnitudes of the instantaneous symmetrical components, an unbalance indicator can also be defined as in Equation (5). The values of such an indicator would be normalized, with 0 indicating perfect balance and 1 indicating that there is no amount of balanced quantities in the three-phase signals around the fundamental frequency.

$$
u_{\underline{x}}(t)=\sqrt{\frac{\left|\underline{x}_{-}(t)\right|^{2}+\left|\underline{x}_{0}(t)\right|^{2}}{\left|\underline{x}_{+}(t)\right|^{2}+\left|\underline{x}_{-}(t)\right|^{2}+\left|\underline{x}_{0}(t)\right|^{2}}}
$$

As previously stated ${ }^{(5)}$, the proposed algorithm implements simple operations and is suited for on-line monitoring. The use of instantaneous symmetrical components enables the possibility to detect changes in the state of the monitored system in real time. More details on the method and algorithm can be found in ${ }^{(5)}$. 


\section{Mechanical faults monitoring}

Mechanical load torque oscillations and shaft dynamic eccentricities experienced by an electromechanical rotating machine induce phase and amplitude modulations in electrical signals measured on this machine ${ }^{(10)}$. For example, bearing faults generating torque oscillations lead to frequency modulations in electrical signals ${ }^{(10,12)}$. Moreover, the characteristic frequency of these modulations can be precisely determined by using the shaft rotating frequency and the bearing geometry. Consequently, bearing faults should be detected by using voltage and current instantaneous amplitude and frequency defined in Equations (3) and (4) where $x$ stands for voltage or current signals.

In order to analyse the performance of this approach, an experimental test-bench developed in the CETIM laboratory in Senlis (France) has been used. This bench, depicted in Figure 1, emulates the structure and behaviour of a wind turbine. The operating conditions are determined by controlling the speed of the low speed shaft, which is considered to be the input of the system. An experiment was conducted for $\approx 200$ hours during which an accelerated deterioration of the main bearing was induced by applying axial and radial forces on the main bearing. At the end of the experiment, inner and outer race faults have occurred in the main bearing. The electrical signals, both three-phase voltages and currents, have been acquired all along this experiment at the stator of the generator. Signals used in this work have been acquired during a stationary operating state of the bench with a constant rotating speed of the low speed shaft of $20 \mathrm{rpm}$. For this rotating speed, the corresponding bearing fault frequencies have been been computed as $B P F O=2.5498 \mathrm{~Hz}$ and $B P F I=3.4502 \mathrm{~Hz}$. The analysis method described in the previous section has been applied to these measurements, and the indicators of Equations (3) and (4) have been computed for both voltage and current sequence components.

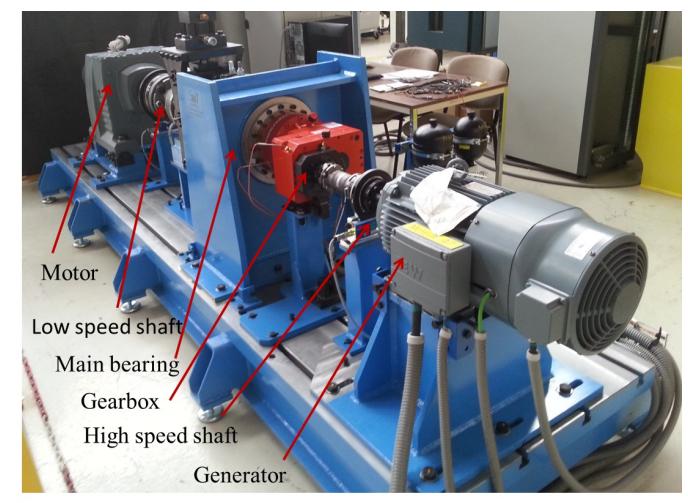

Figure 1: Experimental test bench

Figure 2 presents the power spectral densities (PSDs) estimated for the instantaneous amplitude and frequency of the positive sequence component of the signals using Welch modified periodogram ${ }^{(15)}$. As it can be seen in the figures, the PSDs of all four quantities present a peak at the fault frequency of the inner race of the main bearing in the faulty case. However, the outer race fault is not detectable using these electrical quantities. This limitation might be explained by the physical properties of the system and by the fact that 
the outer race fault does not induce enough torque oscillations to impact the electrical system. The inner race fault, being physically closer to the shaft, does induce torque oscillations which are then propagated through the gearbox and high speed shaft until the electrical generator. The peaks visible in Figure 2 at the frequency marked as BPFI represent the torque oscillations signature in the measured electrical quantities. Moreover, this fault frequency is modulated by the rotating speed of the shaft. The resulting sidebands can also be observed in Figure 2, even though they are less obvious for the PSDs resulting from current quantities.

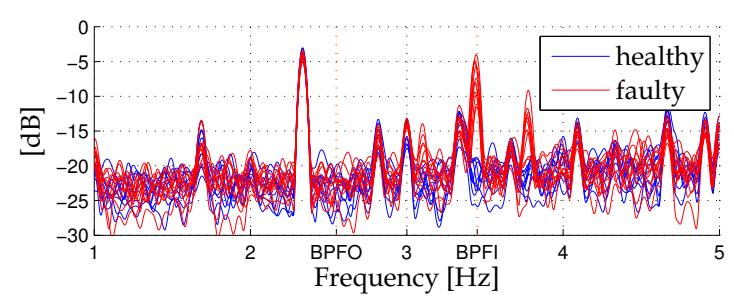

(a) PSD of $\left|v_{+}(t)\right|$

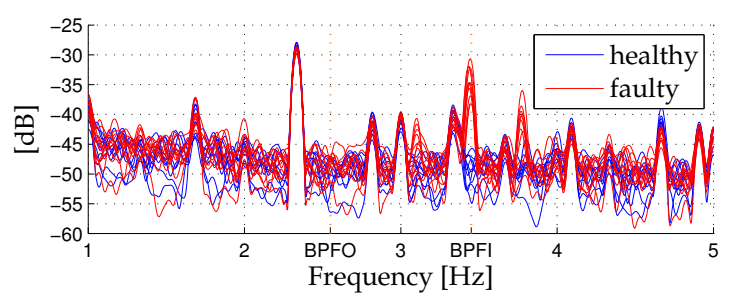

(c) PSD of $f_{0}(t)$ estimated using $v_{+}(t)$

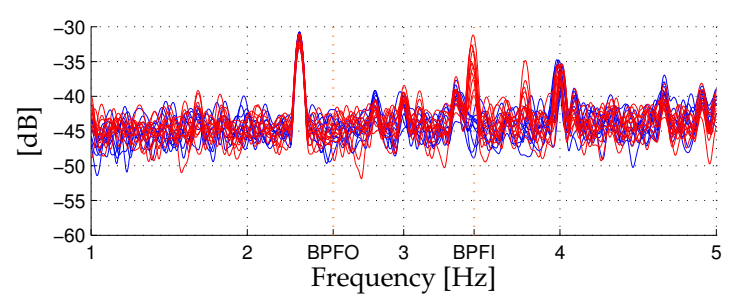

(b) PSD of $\left|i_{+}(t)\right|$

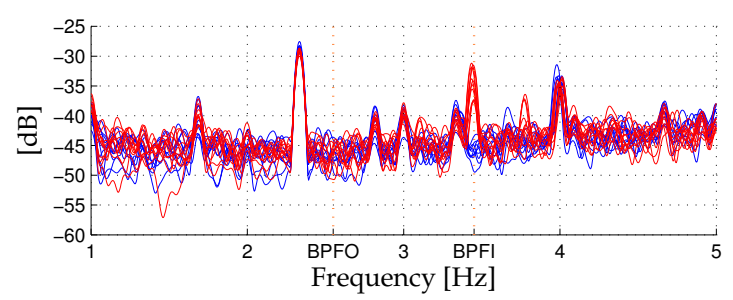

(d) PSD of $f_{0}(t)$ estimated using $i_{+}(t)$

Figure 2: PSDs of the instantaneous amplitude and fundamental frequency of the positive sequence components of the voltage and current

In order to perform automatic fault detection, computing the PSDs is not sufficient and a scalar fault indicator needs to be defined. As previously mentioned, the fault frequency is known thanks to the bearings geometry and the shaft rotating speed. Consequently, the obtained PSDs can be summed up over a frequency band $B$ containing the fault frequency in order to detect eventual faulty components. Equation (6) proposes a normalised version $m_{B}$ of such an indicator:

$$
m_{B}=\frac{\int_{(B)} S(f) \mathrm{d} f}{\int_{(B)} S_{h}(f) \mathrm{d} f},
$$

where $B$ denotes the chosen frequency band, $S_{h}(f)$ denotes a reference PSD obtained in the healthy case and $S(f)$ is the last obtained PSD.

The results obtained for this fault indicator are depicted in Figure 3. Considering that the signals processed for this section have all been acquired in stationary operating conditions and that the number of points used for the Fast Fourier Transform computation is 
known, the peak width is also known. For the spectra depicted in Figure 2, the width of each peak is $0.12 \mathrm{~Hz}$. The results of computing the fault indicator over this peak-wide frequency band are depicted in Figure 3a. Obviously, the fault indicator stays close to 1 in the healthy case and increases in the faulty case. The figure clearly shows that the fault can be detected using either quantity. Moreover, since in case of non-stationarity the peak location and width are not as easily determined, the same indicator has been also computed on a larger frequency band $B=1 \mathrm{~Hz}$ and the results are depicted in Figure $3 \mathrm{~b}$. In this case, using the voltage positive sequence component, the fault can still be detected but at a later time. In the case of using the currents positive sequence component instantaneous amplitude and frequency, the results are less conclusive. Nonetheless, the results seen in Figure 3 show that the proposed indicator is capable of detecting a mechanical fault provided an adequate frequency band is chosen. Moreover, using the PSDs of the instantaneous frequency yields slightly better results than the PSDs of the instantaneous amplitudes for both voltage and current positive-sequence components.

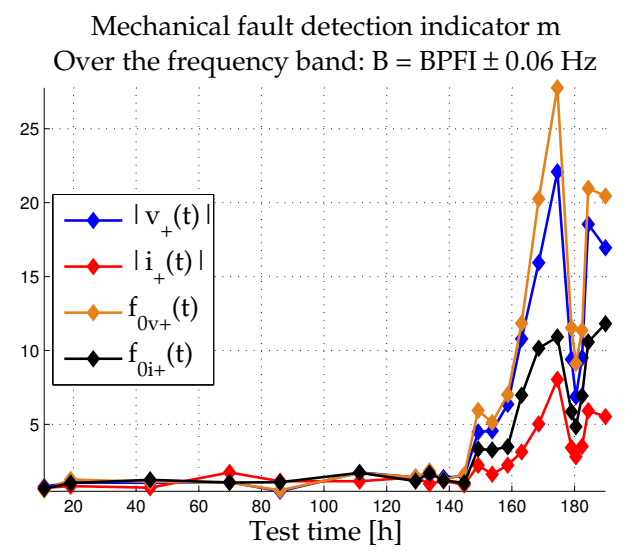

(a) Computed over a peak-wide frequency band

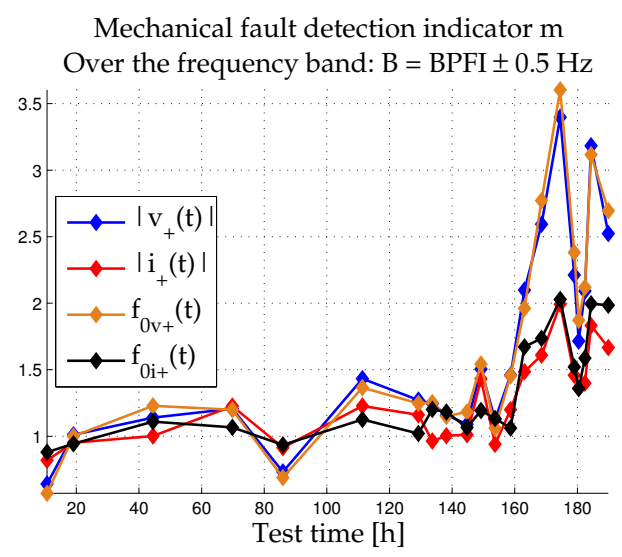

(b) Computed over a $1 \mathrm{~Hz}$ frequency band

Figure 3: Main bearing inner race fault detection (the legends indicate which quantities were used to compute the respective fault indicator)

All in all, the proposed indicator is able to detect the given main bearing inner race fault. Moreover, voltages seem to be better suited for this task and this generator configuration. One possible reason for this behaviour is that they are less influenced by electric loads, compared to the currents. Another explanation is that in the measured signals, the voltages have generally a better signal to noise ratio than the currents around the fundamental frequency.

\section{Electrical faults monitoring}

Winding faults in three-phase electrical systems have been shown to trigger electrical unbalance in three-phase electrical signals ${ }^{(6)}$. Winding faults affect transformers, as well as three-phase electrical rotating machines, whether they operate as a motor or a generator. An increase of the electrical unbalance in such three-phase systems corresponds to an increase in the symmetrical components related to unbalance, i.e. the negative- and the zero-sequence component of the voltages and/or the currents. Consequently, winding 
faults should be detected by using the unbalance indicator defined in Equation (5), where $x$ states for voltage or current signal.

In order to demonstrate the feasibility of this approach in terms of detection of electrical unbalance faults, three-phase voltage signals have been acquired at the output of a transformer. The transformer connection is $\Delta-Y$ (primary being $\Delta$ and secondary being $Y$ connected), and the transformation ratio is $41 / 43$. At the output of the transformer, artificial phase-to-ground faults have been induced through a resistance. Figure 4 depicts the connection for a phase to ground fault on phase 1.

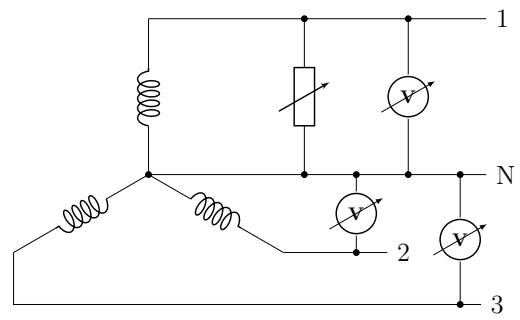

Figure 4: Wye connection phase to neutral fault

The measured electrical signals are phase-to-neutral voltages. Moreover, using the lineto-neutral measured voltages, line-to-line quantities have been computed as in Equations (7).

$$
v_{12}(t)=v_{1}(t)-v_{2}(t) \quad v_{23}(t)=v_{2}(t)-v_{3}(t) \quad v_{31}(t)=v_{3}(t)-v_{1}(t)
$$

The values used for the resistance are depicted in Table 1. Phase to neutral faults have been triggered on each phase respectively, leading to three sets of measurements. Each measurement lasts for $35 \mathrm{~s}$. The first $5 \mathrm{~s}$ are acquired in the absence of the fault and afterwards the resistance value was decreased according to Table 1.

Table 1: Resistance values used for phase-to-ground fault tests

\begin{tabular}{|c|c|c|c|c|c|c|}
\hline Resistance values: & $79.55 \Omega$ & $27.05 \Omega$ & $13.78 \Omega$ & $8.23 \Omega$ & $5.51 \Omega$ & $4.13 \Omega$ \\
\hline in \% of 'open': & 5 & 15 & 30 & 50 & 75 & 100 \\
\hline
\end{tabular}

The proposed electrical unbalance indicator is able to detect the occurrence of such faults, as its definition given by Equation (5) considers the magnitudes of both the negative- and zero-sequence components. Such results are depicted in Figure 5. In Figure 5a, the performance of the indicator is shown for the case when the algorithm was directly applied on the acquired line-to-neutral voltage signals. As shown, the indicator is able to detect and track the evolution of the electrical unbalance occurring in the system. Figure $5 \mathrm{~b}$ depicts the same results, with the difference that the algorithm has been applied on the computed line-to-line voltages. As in this case the zero-sequence component is not available for computation, the obtained magnitude of the indicator is slightly smaller than in the previous scenario. However, fault detection is still possible for all three sets of experiments. 


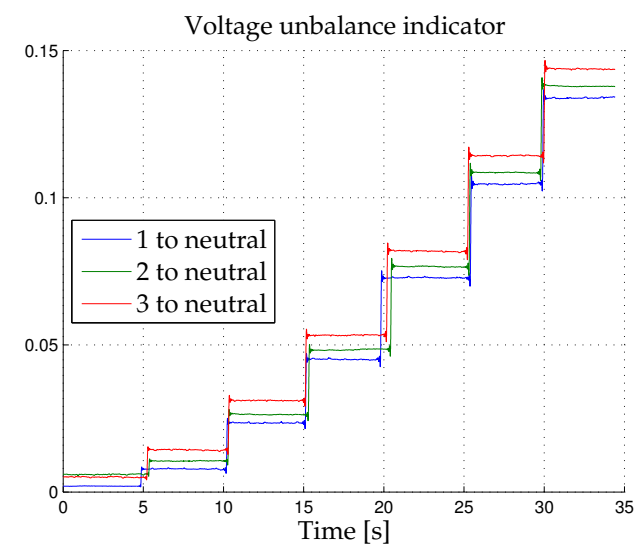

(a) Voltage unbalance using line-to-neutral voltages

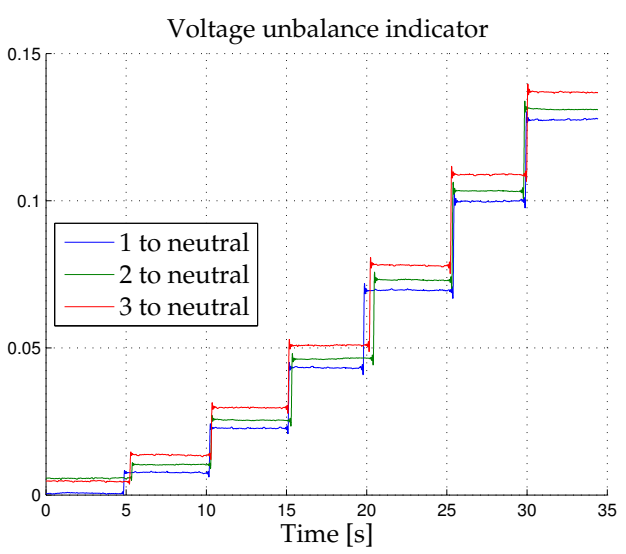

(b) Voltage unbalance using line-to-line voltages

Figure 5: Voltage unbalance indicator results

It has been shown ${ }^{(6)}$ that the angle of the negative- and zero-sequence components can be used to identify the type and phase location of an occurring electrical fault. However, the specific values of the computed angle depend on the electrical system itself and on its configuration and such computations are beyond the scope of the current paper. Nonetheless, the proposed method can also be used to identify the faulty phase where the resistor is connected. As the computed quantities are instantaneous ones, the angle of the negative- and zero-sequence components would depend on time. In order to extract the time-invariant angle of the components, the positive-sequence component can be used as a reference as shown in Equation (8).

$$
a_{-}(t)=\arg \left\{\frac{v_{-}(t)}{v_{+}(t)}\right\} \quad a_{0}(t)=\arg \left\{\frac{v_{0}(t)}{v_{+}(t)}\right\}
$$

The results of applying these computations are depicted in Figures $6 \mathrm{a}$ and $6 \mathrm{~b}$. It can be observed in the figures that the angles of the considered components depend on the phase on which the unbalance occurs. Moreover, while the amplitudes of the ratios used in (8) increase with the fault severity, the computed angles remain relatively constant in time. The angles computed for each type of fault are separated by a phase shift of $\frac{2 \pi}{3}$ and can be clearly used to identified the faulty phase.

If line-to-line quantities are used, the information on the zero-sequence component is not available. However, the angle of the negative-sequence component can still be obtained and its value is related to the one obtained using the line-to-neutral measurements. Considering the property of the Fortescue transformation to split any unbalanced N-phase system into $\mathrm{N}$ balanced systems, the symmetrical components from line-to-neutral and line-to-line measurements are related by a magnitude of $\sqrt{3}$ and an angle of $30^{\circ}$. The relation between these two quantities is given by Equation (9). These results are depicted in Figure 6c.

$$
a_{-L L}(t)=\arg \left\{\frac{v_{-L L}(t)}{v_{+L L}(t)}\right\}=\arg \left\{\frac{\sqrt{3} v_{-L N}(t) e^{-j 30^{\circ}}}{\sqrt{3} v_{+L N}(t) e^{j 30^{\circ}}}\right\}=a_{-}(t) e^{-j 60^{\circ}}
$$


This result is confirmed by figures $6 \mathrm{a}$ and $6 \mathrm{c}$ where the phase shift of $-60^{\circ}$ between the two cases is clearly visible.

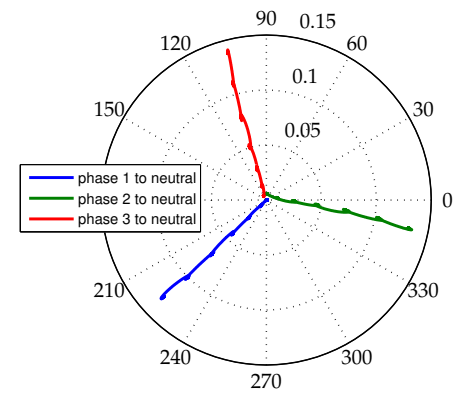

(a) Angles between negativeand positive-sequence line-to-neutral voltages

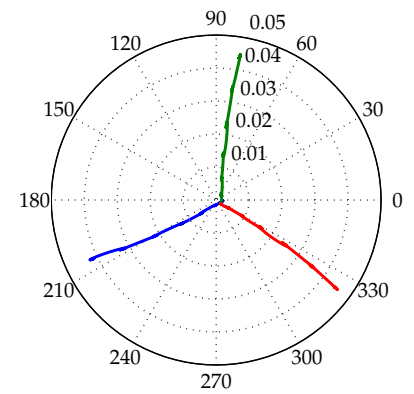

(b) Angles between zeroand positive-sequence line-to-neutral voltages

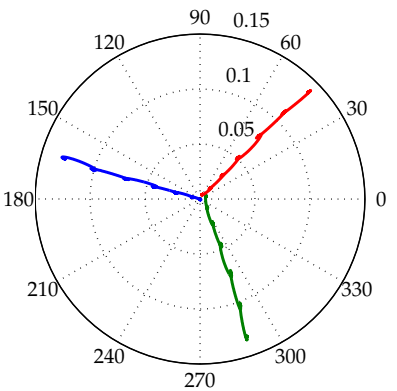

(c) Angles between negativeand positive-sequence line-to-line voltages

Figure 6: Preliminary results in fault localisation

All in all, it has been shown that the proposed algorithm for on-line condition monitoring of electrical three-phase systems is capable of detecting electrical unbalance in such systems. Empirically, it has also been shown that fault localisation is possible using the method proposed in this section. However, in order to correctly identify the phase(s) on which the unbalance occurs, angle computations have to be done for each system specifically.

\section{Wind turbine continuous monitoring}

The algorithm proposed in this paper has been implemented on an embedded system developed for the KAStrion project. This system has been installed on two wind turbines (WT1 and WT2), with the courtesy of VALOREM, located in an onshore wind farm in the south of France. In this section, preliminary results obtained by the three-phase electrical analysis method are presented using the signals acquired on the two wind turbines. The considered variable speed wind turbines have a rated power of $2 \mathrm{MW}$ and are equipped with asynchronous generators. The acquired electrical signals are three-phase voltages and currents at the stator of the generator.

The signals used in this paper have been acquired from mid-December 2014 until midJanuary 2015, on both wind turbines. The signal samples have been recorded over $10 \mathrm{~s}$ and they belong to several operating states of the wind turbine, for a wind speed varying between 5 to $20 \mathrm{~m} . \mathrm{s}^{-1}$ and a rotating speed of the high speed shaft between 1000 and $1800 \mathrm{rpm}$. Figure 7 depicts the results obtained on the wind turbine signals in terms of electrical unbalance around the fundamental frequency. As a reminder, the electrical unbalance indicator is a normalised quantity belonging to the interval $[0,1]$, with 0 representing perfect balance. Moreover, in order to present the behaviour of the instantaneous indicators over a longer period of time, they have been characterized by their mean value and standard deviation. The electrical unbalance values depicted in Figure 7 for the 
voltages represent the mean value of the instantaneous quantity.

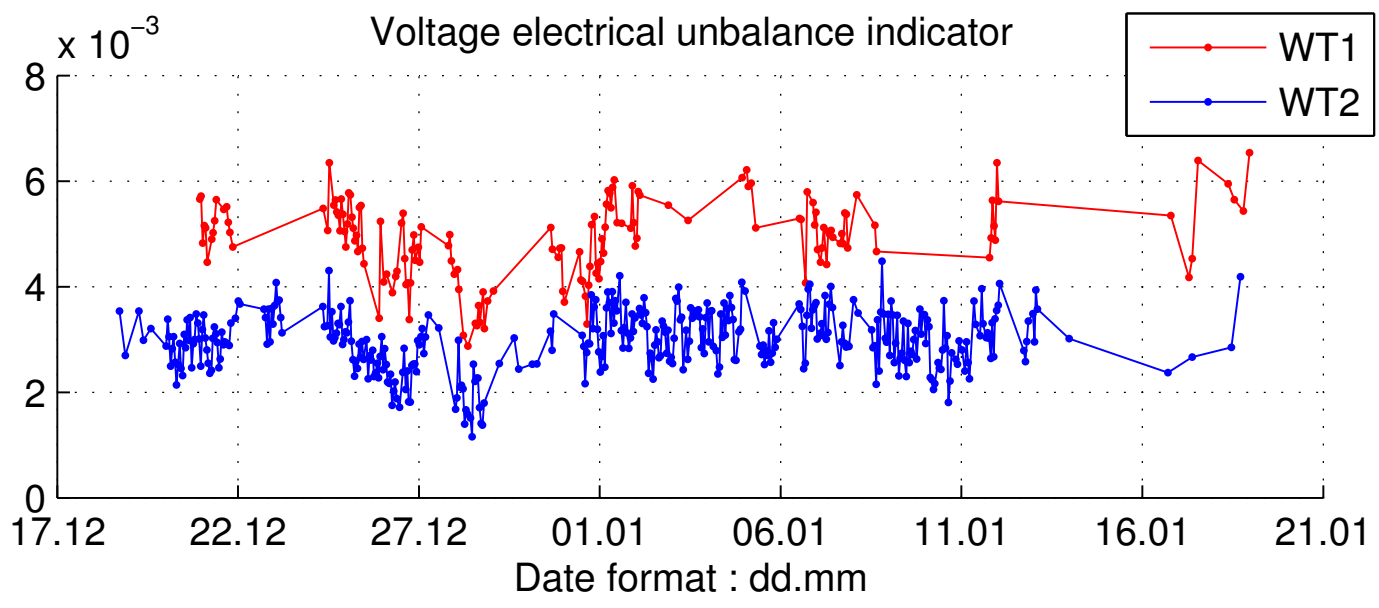

Figure 7: Electrical unbalance evolution in two monitored wind turbines

In Figure 7 it can be observed that the average values of the electrical unbalance indicators do not seem to be very affected by the operating state of the wind turbines. Moreover, these indicators have a constant trend for this one month time period, suggesting that no increase in the amount of electrical unbalance occurred in these two systems. The voltage signals contain a very small amount of electrical unbalance, most probably due to the inherent unbalance present in any real three-phase electrical system. However, this value is really small, to the order of $10^{-3}$.

Considering that there is no a priori knowledge of the healthy state of the generators mounted in the wind turbines, it is difficult to estimate the correct amount of expected natural electrical unbalance. Nonetheless, by using the condition monitoring system thus far developed within the KAStrion project framework, the evolution of the system state can now be monitored online.

\section{Conclusions}

In the first part of this paper, the proposed online algorithm for three-phase electrical signals analysis has been briefly presented. Following, the algorithm has been tested on mechanical fault detection and corresponding results have been presented. It has been shown that mechanical faults can be detected using the electrical quantities measured at the output of the generator. However, certain limitations do apply: the mechanical faults have to induce torque variations, which in turn have to propagate through the mechanical system. Moreover, the fault frequency has to be within the frequency band used by the complex valued filter applied on the electrical signals. Nonetheless, the filter frequency band is a configurable parameter of this method.

In terms of electrical unbalance, the proposed method has been applied on experimental signals obtained on a laboratory test-bench using a three-phase transformer. It has 
been shown that electrical unbalance can be detected and tracked in real time using the proposed method. Moreover, under the assumption that system-specific electrical computations are performed, the faulty phase can be identified using the angle of the negativeand zero-sequence components with respect to the positive one. While the theoretical value for each angle has not been computed for this paper, the results presented do show that the obtained angle values depend on the faulty phase and that the three obtained angles (for three different phase to neutral faults) are separated by a phase shift of $\frac{2 \pi}{3}$ and can be used to localized the faulty phase.

In the last section of this paper, results of continuous condition monitoring of two onshore wind turbines have been presented. During the considered one month time period, a relatively constant value for the voltage electrical unbalance has been observed for both wind turbines, regardless of the wind turbines operating states. The small constant value of the unbalance is considered to be due to the natural unbalance of the system. However, no a priori knowledge of the healthy state of the system is available and until the time this paper has been submitted, there has been no inspection of either turbine to confirm the findings. Nonetheless, over a time period of roughly one month, no clear evolution of the health state of the two wind turbines has been observed from the electrical point of view.

\section{Acknowledgements}

This research has been partly supported by KIC InnoEnergy, a company supported by the European Institute of Innovation and Technology (EIT), through KAStrion European project.

The authors would like to thank CETIM (Centre Technique des Industries Mécaniques) for providing the test-bench and experimental data.

The authors would also like to thank VALEMO (a subsidiary of VALOREM) for installing the KAStrion prototype of the embedded software on the two wind turbines.

\section{References}

1. European Wind Energy Association (EWEA), 'Wind in power: 2014 European statistics', Available online:

http://www.ewea.org/fileadmin/files/library/publications/statistics/EWEA-AnnualStatistics-2014.pdf, Feb. 2015.

2. KAStrion project official web-site: http://www.gipsa-lab.fr/projet/KASTRION/

3. G. Song, Z.-Y. Li, P. Bellemain, N. Martin and C. Mailhes, 'AStrion data validation of nonstationary wind turbine signals', CM2015 \& MFPT2015, June 2015.

4. Z.-Y. Li, T. Gerber, M. Firla, P. Bellemain, N. Martin and C. Mailhes, 'AStrion strategy: from acquisition to diagnosis. Application to wind turbine monitoring', CM2015 \& MFPT2015, June 2015. 
5. G. Cablea, P. Granjon, and C. Bérenguer, 'Method for computing efficient electrical indicators for offshore wind turbine monitoring', Insight - Non-Destructive Testing and Condition Monitoring, vol. 56, no. 8, pp. 443-448, Aug. 2014.

6. M. B. K. Bouzid and G. Champenois, 'New Expressions of Symmetrical Components of the Induction Motor Under Stator Faults', IEEE Transactions on Industrial Electronics, vol. 60, no. 9, pp. 4093-4102, Sep. 2013.

7. W. V. Lyon, 'Transient analysis of alternating current machinery - an application of the method of symmetrical components', The Technology Press of MIT and John Wiley \& Sons, New York, 1954.

8. G. C. Paap, 'Symmetrical components in the time domain and their application to power network calculations', IEEE Transactions on Power Systems, vol. 15, no. 2, pp. 522-528, May 2000.

9. C. L. Fortescue, 'Method of Symmetrical Co-Ordinates Applied to the Solution of Polyphase Networks', Transactions of the American Institute of Electrical Engineers, vol. XXXVII, no. 2, pp. 1027-1140, Jul. 1918.

10. M. Blodt, P. Granjon, B. Raison, and J. Regnier, 'Mechanical Fault Detection in Induction Motor Drives through Stator Current Monitoring - Theory and Application Examples', in Fault Detection, W. Zhang, Ed. InTech, 2010.

11. P. Granjon, 'Condition monitoring of motor-operated valves in nuclear power plants', in: 8th International Conference on Condition Monitoring and Machinery Failure Prevention Technologies, Cardiff, UK, vol. 1, pp 109-119, 2011.

12. V. Choqueuse, M. E. H. Benbouzid, Y. Amirat, and S. Turri, 'Diagnosis of ThreePhase Electrical Machines Using Multidimensional Demodulation Techniques', IEEE Transactions on Industrial Electronics, vol. 59, no. 4, pp. 2014-2023, Apr. 2012.

13. A. Reilly, G. Frazer and B. Boashash, 'Analytic signal generation âĂŞ tips and traps', IEEE Transactions on Signal Processing, vol. 42, no. 11, pp. 3241-3245, Nov. 1994.

14. B. Boashash, 'Estimating and interpreting the instantaneous frequency of a signal. I. Fundamentals', Proceedings of the IEEE, vol. 80, no. 4, pp. 520-538, Apr. 1992.

15. Welch P., 'The use of fast Fourier transform for the estimation of power spectra: A method based on time averaging over short, modified periodograms', IEEE Transactions on Audio and Electroacoustics, vol. 15, pp. 70-73, 1967. 